Gut, 1967, 8, 253

\title{
Peptic ulceration and gastrointestinal bleeding in pancreatitis
}

\author{
I. N. MARKS, S. BANK, J. H. LOUW, AND JACK FARMAN \\ From the Gastro-Intestinal Service, Groote Schuur Hospital, and the Departments of Medicine, \\ Surgery and Diagnostic Radiology of the University of Cape Town, Observatory, \\ Cape Town, South Africa
}

EDITORIAL COMMENT Acute gastrointestinal bleeding has been present in $20 \%$ of this large series but in only a few of these can it be attributed to chronic peptic ulceration. The wide variety of causes is commented upon in this paper.

Radiological evidence of peptic ulceration is not unusual in patients with pancreatitis. This, coupled with the not infrequent occurrence of gastrointestinal bleeding in pancreatitis, has led many to believe that a high incidence of peptic ulceration exists in patients with this condition (Warren and Cattell, 1959; Fitzgerald, Fitzgerald, Fennelly, McMullin, and Boland, 1963; Howard and Jordan, 1960; Zollinger, Elliot, Endahl, Grant, Goswitz, and Taft, 1962; Eyler, Clark, and Rian, 1962). Others, on the other hand, have reported a low incidence of ulceration in patients with pancreatitis (Marks and Bank, 1963).

The purpose of the present paper is to re-examine the incidence of peptic ulceration in pancreatitis, to draw attention to the fact that the radiological changes in pancreatitis may mimic peptic ulceration, and to analyse the causes of gastrointestinal bleeding in patients with pancreatic disease.

\section{MATERIAL}

The patients studied were referred to the GastroIntestinal Service from the medical, surgical, and paediatric divisions at the Groote Schuur and associated hospitals during the five-year period 19601965. Many were known to have pancreatic disease at the time of referral, but the majority were investigated because of symptoms suggestive of pancreatitis or as part of the routine investigations for steatorrhoea or cirrhosis. The series comprised 529 patients with pancreatitis (Table I). Alcohol was the major aetiological factor in 336, and biliary disease in 80 . Pancreatitis due to penetrating peptic ulcers accounted for nine cases, and other miscellaneous causes for 59. No cause could be ascertained in the remaining 45 patients.

Calcification of the pancreas was present in 85 of the
TABLE I

CLINICAL CLASSIFICATION OF PATIENTS WITH PANCREATITIS

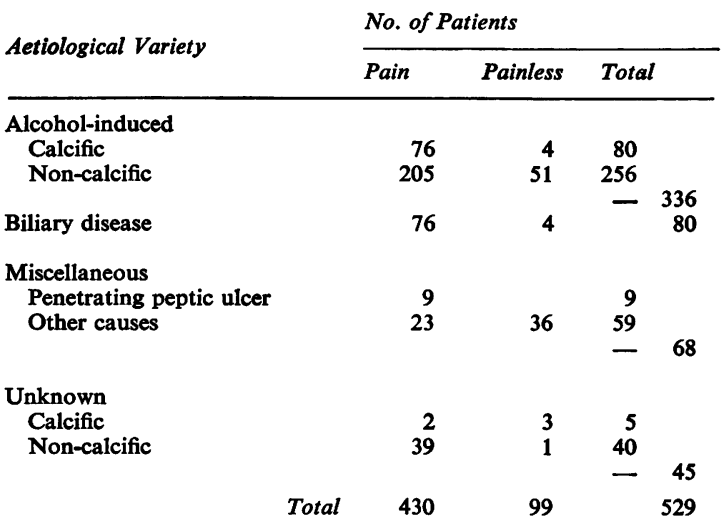

529 patients; 99 were considered to have pancreatic disease unassociated with pain. Pancreatic disease was diagnosed by means of surgery, the presence of pancreatic calcification on radiographs of the abdomen, or by the finding of an abnormality in the secretin-pancreozymin pancreatic function test (Bank, Marks, Moshal, Efron, and Silber, 1963). Pancreatic disease was also diagnosed in patients with a strongly suggestive story of pancreatitis in whom two or more of the following biochemical abnormalities were present: a borderline pancreatic function test, an elevated serum amylase level, a positive provocative enzyme test, or an abnormal glucose tolerance test.

Barium meal examinations were carried out as a routine procedure in patients with calcific pancreatitis and in all patients with persistent pain or dyspepsia. An augmented histamine test was also carried 
out in the majority of such patients. Many patients with radiological evidence of peptic ulceration were subjected to laparotomy and in some the suspect area in the stomach or duodenum was visualized by means of gastrotomy or pyloroplasty respectively. Particular attention was paid to the clinical story and the response to intensive ulcer therapy in all patients with radiological evidence of ulceration, especially in those in whom laparotomy was not carried out.

Patients with gastrointestinal bleeding were interrogated with special reference to their intake of alcohol, the time interval between the onset of bleeding and the previous alcoholic bout, the relationship of the onset of the bleeding to the clinical onset of pancreatitis, and the presence or absence of severe retching and vomiting immediately before the onset of the haematemesis. The patients were also questioned with regard to the presence of fresh or altered blood in the vomitus. Routine biochemical tests for cirrhosis were carried out in all patients, and the majority had liver biopsies. Barium meal examination was carried out in all patients, and an augmented histamine test and gastroscopy was also performed on most of them. Laparotomy was carried out when indicated. Portal splenography was not undertaken.

\section{RESULTS}

PATIENTS WITH RADIOLOGICAL EVIDENCE OF PEPTIC ULCERATION OR CARCINOMA Radiological evidence of peptic ulceration or carcinoma was present in 48 patients (Table II). Nine of the 48 patients gave a history of periodic ulcer dyspepsia and were found, on testing or at laparotomy, to have evidence of pancreatic disorder. Laparotomy was carried out in seven of the nine patients and revealed a posterior penetrating gastric or duodenal ulcer, with a localized area of pancreatitis surrounding the base of the ulcer. In this small group no difficulty was experienced in differentiating an indurated head of the pancreas secondary to duodenal ulceration from a carcinoma of the head of the pancreas. Only 14 of the remaining 39 patients were considered to have a peptic ulcer and one other had bled from a gastric cancer. Of the 14 patients, five were found to have a gastric ulcer and four a duodenal ulcer at laparotomy. Theclinical story, response to alkalis, and high acid output were strongly suggestive of a duodenal ulcer in the five remaining patients, one of whom may have had pancreatitis secondary to ulcer penetration.

The radiological appearance suggestive of peptic ulceration or carcinoma of the stomach was considered to be due to pancreatic swelling or peripancreatic inflammation in 24 of the 48 patients (Table II). These comprised five with radiological changes simulating gastric ulceration, three regarded as being due to gastric carcinoma, and 16 with an appearance mimicking duodenal ulceration or scarring. Laparotomy disproved the presence of ulceration in two of the five patients with alleged gastric ulceration and in the remaining three the absence of an ulcer type dyspepsia, failure of alkalis to influence the persistent pain, and normal gastroscopic findings pointed against the radiological diagnosis. However, none of the five patients showed a typical mid-lesser curve ulcer niche. Two patients with suspected gastric carcinoma showed antral irregularity and mucosal distortion, and a third patient had a filling defect on the lesser curve aspect on the barium meal examination; an intrinsic gastric lesion was excluded by laparotomy in all three patients.

The 16 patients with false-positive ulceration or scarring of the duodenal cap on barium meal examination were of interest in that a confident preoperative diagnosis of active duodenal ulceration was made in no fewer than seven of 10 patients in this group submitted to operation. The radiographic appearances in these patients were so compelling that the presence of persistent rather than an ulcertype pain, the failure to achieve a therapeutic response with intensive alkali medication, and, in some, the finding of pancreatic calcification, mild

TABLE II

RADIOLOGICALLY DEMONSTRABLE PEPTIC ULCERATION OR CARCINOMA

\begin{tabular}{|c|c|c|c|c|c|c|}
\hline \multirow[b]{2}{*}{ Diagnosis } & \multicolumn{3}{|c|}{ Positive Group } & \multicolumn{3}{|c|}{ False Positive Group } \\
\hline & $\begin{array}{l}\text { Laparotomy } \\
\text { Evidence }\end{array}$ & $\begin{array}{l}\text { Clinical and } \\
\text { Biochemical } \\
\text { Evidence }\end{array}$ & Total & $\begin{array}{l}\text { Laparotomy } \\
\text { Evidence }\end{array}$ & $\begin{array}{l}\text { Clinical and } \\
\text { Biochemical } \\
\text { Evidence }\end{array}$ & Total \\
\hline
\end{tabular}

Penetrating gastric ulcer

Penetrating duodenal ulcer

$\begin{array}{ll}3 & 0 \\ 4 & 2 \\ 1 & \\ 5(2)^{1} & 0 \\ 4(2) & 5^{2}\end{array}$

Carcinoma stomach

Gastric ulcer + pancreatitis

Duodenal ulcer + pancreatitis

3(1)

$10(4)$

24

${ }^{1}$ Figures in parentheses represent number of patients with calcific pancreatitis.

${ }^{2}$ Includes one patient in whom the pancreatitis may have been secondary to a penetrating duodenal ulcer. 


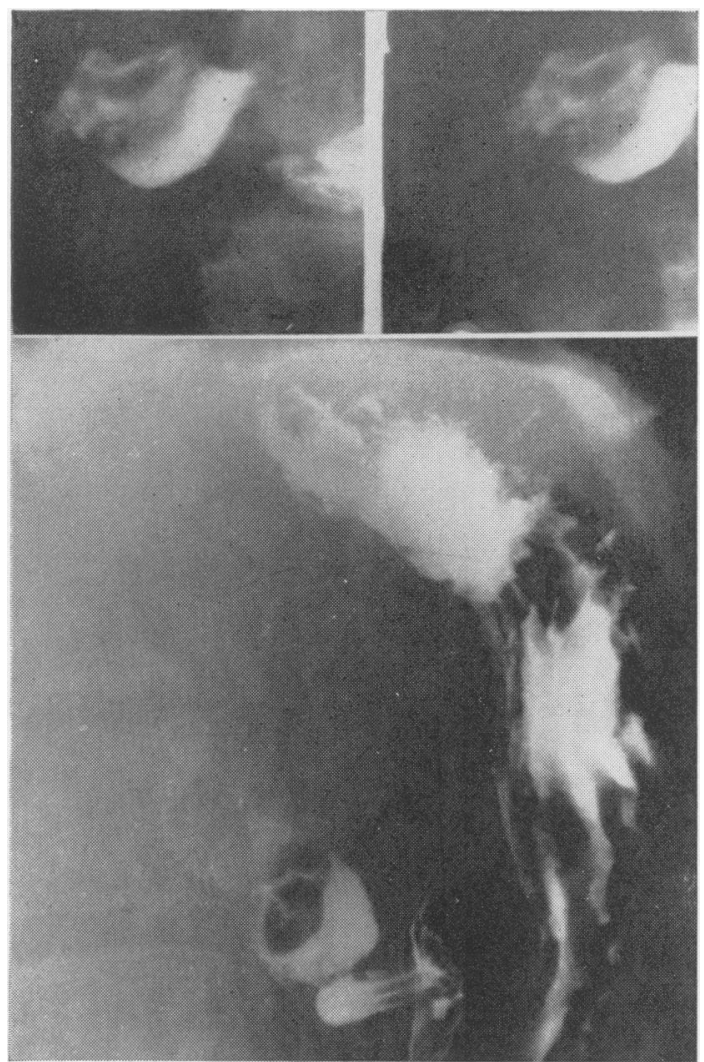

FIG. 1. icterus, and virtual achlorhydria were disregarded or overlooked in favour of the radiological diagnosis. The diagnosis of duodenal ulcer or scarring was refuted by surgery in the 10 patients referred for laparotomy, and considered most unlikely in the remaining six in view of the lack of an ulcer-type dyspepsia, absence of a response to alkalis, and normal or low levels of acid secretion.

Two types of duodenal deformity were encountered in these 16 patients, the first manifesting as a rounded filling defect containing a central barium collection, the 'target deformity,' and the second as a deformity strongly resembling that due to the scarring of peptic ulceration. The 'target deformity' was interpreted as being due to a large ulcer niche with surrounding oedema (Figs. 1 and 2) but we now appreciate that it represents a compression defect strongly suggestive of pancreatic disease (Farman, Werbeloff, Marks, Bank, and Louw, 1966).

PATIENTS WITH ULCER-TYPE DYSPEPSIA WITH NO RADIOLOGICAL EVIDENCE OF ULCERATION AND CONSIDERED TO BE DUE TO PANCREATIC DISEASE There were nine patients recorded as having a history suggestive of peptic ulceration in whom the barium meal examination revealed no ulcer. The response to ulcer treatment in these patients was unsatisfactory, and the results of the augmented histamine test and gastroscopy contributed nothing. Laparotomy in four of the nine patients showed the presence of pancreatic disease

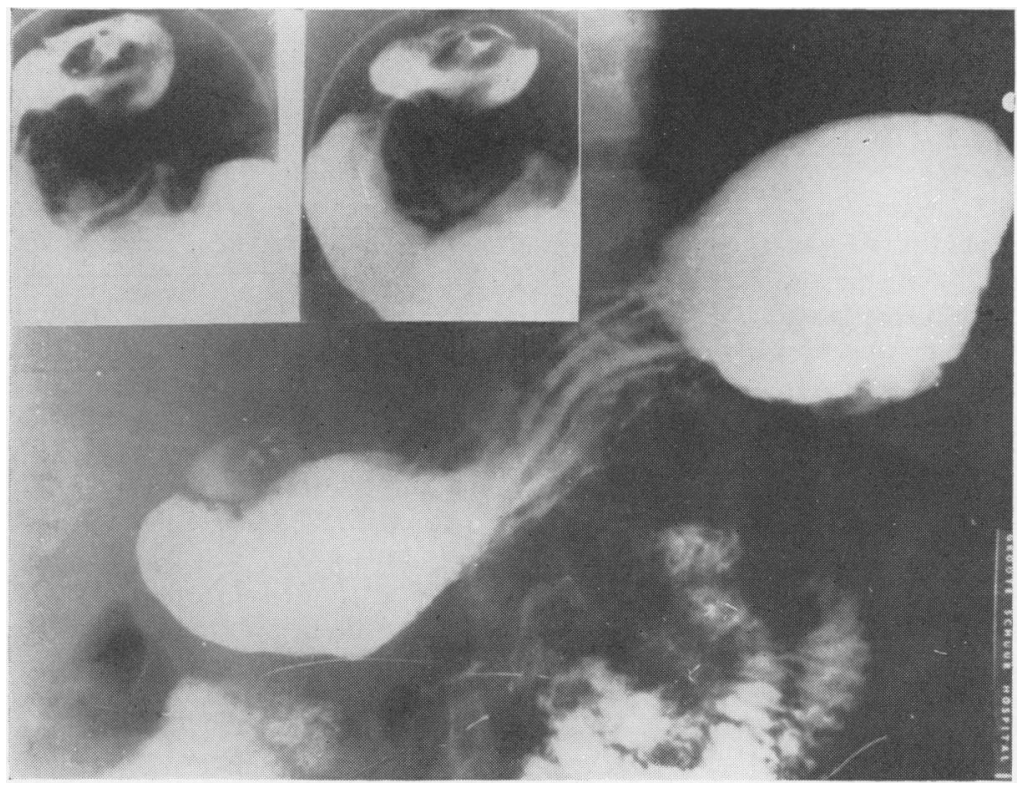

FIG. 1. Barium meal in case 1 showing filling defect in duodenal cap with central barium collection due to pancreatic disease. These changes were interpreted as being due to an active duodenal ulcer in the posterior wall of the cap with surrounding oedema.

FIG. 2. Barium meal in case 2 showing filling defect in duodenal cap with central barium collection and apparent niche with radiating folds in pyloric antrum. These changes were due to pancreatic disease associated with cyst formation in the head of the pancreas. 
and confirmed the absence of peptic ulceration; two of these patients had a cyst in the head of the pancreas. A strong history of alcoholism and an abnormal pancreatic function test was present in the remaining five patients, three of whom showed marked acid hyposecretion. Heartburn was not a feature in patients with 'pancreatic dyspepsia'. Postprandial attacks of gallstone colic in one of the patients in the series were misinterpreted as an ulcer dyspepsia. Barium meal was negative, and laparotomy for a subsequent attack of gallstone pancreatitis showed no evidence of ulceration.

GASTROINTESTINAL BLEEDING IN PATIENTS WITH PANCREATIC DISEASE Gastrointestinal bleeding occurred in 40 patients, one of whom bled on two separate occasions from different causes. A further five of the 40 patients had more than one episode of haemorrhage but the evidence in these pointed to a similar cause being responsible in each patient. For the purpose of this presentation each of these five patients was considered to have had only one episode of bleeding. The majority of patients had both haematemesis and melaena, but a few had one or the other.

An analysis of the causes of the 41 gastrointestinal haemorrhages is presented in Table III.

HAEMORRHAGE UNRELATED TO AN ATTACK OF PANCREATITIS (13 PATIENTS) The haemorrhage was due to peptic ulceration in six patients, to carcinoma of the stomach in one and, in three patients with painless pancreatic disease, to oesophageal varices associated with cirrhosis of the liver. Two patients with a recent story of early morning anorexia developing against a background of alcoholic insult had an episode of bleeding, presumably on the basis of alcoholic gastritis. In one patient the cause of an episode of painless melaena, followed a few months later by anaemia due to occult bleeding, could not be ascertained.

HAEMORRHAGE ASSOCIATED WITH AN ATTACK OF PANCREATIS (22 PATIENTS) The majority of patients had an obvious attack of alcohol-induced pancreatitis at the time of the bleed. Haemorrhage was the most arresting feature in a number of patients, however, and in these abdominal pain was frequently unimpressive. Indeed, the clinical diagnosis of pancreatitis in a few patients was suggested by the presence of low-grade fever, transient glycosuria and biochemical jaundice, or elevation of the serum amylase level rather than by the abdominal pain.

An alcoholic with a recent history of early morning anorexia and nausea had a haematemesis on the morning following a night's overindulgence. The bleed was followed, a few hours later, by the onset of an attack of pancreatitis. There was a past history
TABLE III

GASTROINTESTINAL BLEEDING IN PATIENTS WITH PANCREATIC DISEASE

$\begin{array}{lll}\text { Cause of Bleed } & \begin{array}{l}\text { Proved by } \\ \text { Laparotomy }\end{array} & \begin{array}{l}\text { Clinical and } \\ \text { Radiological } \\ \text { Evidence }\end{array}\end{array} \quad$ Total

Bleed Unrelated to Attack of Pancreatitis

Duodenal ulcer

Gastric ulcer

Carcinoma stomach

Cirrhosis with varices

? Alcoholic gastritis

Unknown

2
3
1
$2^{3}$
0
0

$\begin{array}{lr}1 & 3^{1} \\ 0 & 3^{1} \\ 0 & 1^{1} \\ 1 & 3^{2} \\ 2 & 2 \\ 1 & 1 \\ & 13\end{array}$

Bleed Associated with Attack of Pancreatitis

$\begin{array}{lllr}\text { ? Alcoholic gastritis } & 0 & 1 & 1 \\ \text { Mallory-Weiss syndrome } & 1 & 2 & 3 \\ \text { Mucosal changes due to } & & & \\ \text { contiguous pancreatitis } & 2^{3} & 0 & 2 \\ \text { ? Splenic vein obstruction } & 2 & 0 & 2 \\ \text { No cause established } & 5\left(1^{8}\right)\left(1^{1}\right) & 9^{4} & 14 \\ & & & 22\end{array}$

Bleed Attributable to Pancreatic Surgery or Investigation

(a) Erosion of blood vessel in cyst wall following: Drainage of cyst Biliary surgery (postoperative cyst)

$2^{3}$

$1^{3}$

3

(b) Following:

$T$-Tube drainage and sphincterectomy

Unsuccessful jejunal biopsy

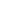

$\mathbf{0}$$$
\text { biopsy }
$$

$\begin{array}{lll}0 & 1 & 1 \\ 0 & 1 & 1 \\ & & 6\end{array}$

${ }^{1}$ Eight patients with radiological evidence of peptic ulceration or carcinoma

'Painless pancreatic disorder

${ }^{3}$ Necropsy evidence

One of these patients bled post-operatively.

of typical attacks commencing some 24 to 48 hours after an alcoholic bout. A barium meal showed no abnormality. The story of bleeding 'on the morning after the night before' suggested alcoholic gastritis as a probable cause in this patient.

Three patients gave a history of profound nausea and vomiting culminating in the vomiting up of fresh blood. The vomiting and haematemesis occurred immediately before the onset of the clinical attack in one of the patients and shortly after the onset of the attack in the remaining two. Laparotomy evidence for the diagnosis of the Mallory-Weiss (Mallory and Weiss, 1929; editorial British Medical Journal 1965) syndrome was obtained in one, and the diagnosis in two was based on the history and negative barium meal findings.

The bleed could be attributed to acute gastric erosion or mucosal congestion secondary to contiguous pancreatitis in two patients. One suffered a lethal haemorrhage during the course of an attack of idiopathic pancreatitis, and another had a haemorrhage during the course of a fulminating attack of 
alcohol-induced pancreatitis. Necropsy in the former showed the posterior wall of the stomach to be adherent to a markedly oedematous and necrotic pancreas; a superficial gastric ulcer about $1 \mathrm{~cm}$. in diameter and several petechiae were found in the region of the adherent stomach and a small, open blood vessel was demonstrated in the base of the ulcer. Necropsy in the second patient revealed intense mucosal congestion and a large submucosal haematoma in the third part of the duodenum adjacent to the acutely inflamed head of pancreas.

Gastro-oesophageal varices associated with splenic vein obstruction was considered to be responsible for bleeding occurring during the course of an attack of alcohol-induced pancreatitis in two patients with splenomegaly. Neither of these patients had clinical, biochemical, or histological evidence of cirrhosis of the liver. The bleed in one of the patients was heralded by the rapid development of splenomegaly during the course of an attack, and the other had had four identical episodes of massive haematemesis and melaena occurring two to four days after an alcoholic bout and following the onset of a clinical attack of pancreatitis. Laparotomy confirmed the presence of chronic pancreatitis and splenomegaly in each case and showed no evidence of cirrhosis or peptic ulceration; splenectomy was carried out. Both patients subsequently had further attacks of alcohol-induced pancreatitis but neither suffered a further bleed. The evidence pointed strongly to a diagnosis of bleeding due to splenic vein compression but the absence of portal splenographic data precluded a firm diagnosis.

The cause of the bleeding could not be established in 14 patients, four of whom had laparotomy. Peptic ulceration, gastric cancer, and portal hypertension due to cirrhosis could be excluded in all. The bleeding may have been due to salicylates in three patients and, in the remainder, the time relationship of the onset of bleeding to the commencement of the attack suggested that the causes cited above may well have been responsible.

Bleeding from a chronic peptic ulcer or from portal hypertension associated with cirrhosis was not found in any of the 22 patients in this group. Only one patient in the group died from an exsanguinating haemorrhage, and a further two died from the associated pancreatitis rather than from the effects of blood loss. Of the 22 patients, 11 required blood transfusion ranging from 600 to $5,400 \mathrm{ml}$.

BLEEDING ATTRIBUTED TO PANCREATIC SURGERY OR INVESTIGATIONS The postoperative period was complicated by massive haematemeses and melaena in three patients following internal drainage of a pancreatic cyst into the stomach or jejunum, and in another patient, who developed a pseudo-cyst of the pancreas following cholecystectomy and T-tube drainage of the common bile duct for gallstone pancreatitis. Three of these patients died from blood loss; the necropsy data indicated that the bleeding was due to erosion of a blood vessel in the wall of the cyst in each of these patients. One of the four patients survived the bleed. Another patient had haematemesis and melaena on the ninth postoperative day following cholecystectomy, duodenotomy, and sphincterotomy for relapsing pancreatitis of unknown cause. One patient with calcific pancreatitis had a massive bleed within 24 hours of an unsuccessful peroral jejunal biopsy but the site of the bleeding could not be ascertained at laparotomy four days later.

\section{DISCUSSION}

Radiological evidence of peptic ulceration or carcinoma and gastrointestinal haemorrhage was found in 77 of $\mathbf{4 3 0}$ patients with painful pancreatitis reviewed in the present study. This incidence $(18 \%)$ appeared to be in keeping with the generally accepted incidence of $15 \%$ to $19 \%$ (Warren and Cattell, 1959; Fitzgerald et al., 1963; Howard and Jordan, 1960; and Zollinger et al., 1962), of peptic ulceration in pancreatic disease. One of the patients had a carcinoma of the stomach, but the diagnosis of gastric or duodenal ulceration could be substantiated in only 23 of the remaining 76 patients. Our incidence of $5.3 \%$ for peptic ulceration was comparable to that of $7 \%$ reported by Boyer and MacKay (1960). It should be stressed, however, that even these figures probably represent an overestimate of the true incidence of the chance association of peptic ulceration and pancreatitis because they include patients with pancreatitis secondary to posterior penetration of a peptic ulcer. There were nine such patients in the present series, leaving a mere $14(3.5 \%)$ in whom peptic ulceration and clinical attacks of pancreatitis were a seemingly chance association. This low incidence was in keeping with our earlier impression regarding the low incidence of peptic ulceration in pancreatitis (Marks and Bank, 1963) and was, in fact, considerably lower than the generally accepted figure of 15 to $19 \%$.

One can only speculate on the possible causes of the discrepancy between the low incidence of peptic ulceration found in the present study and the rather high incidence reported by most previous workers. (a) Farman et al. (1966), in a recent review of duodenal cap deformities in pancreatitis, have drawn attention to the two types of deformity which may simulate deformities due to active or chronic duodenal ulceration (Lubert and Krause, 1963; 
Konstam, 1963; Comfort, Gambill, and Baggenstoss, 1946; Blumenthal and Probstein, 1959; Gambill, 1960; Berk, 1955). The first, a rounded filling defect of the cap containing a central barium collection and described as a 'target deformity,' is due to posterior encroachment on the cap by the irregular surface of the swollen pancreas. The second type closely resembles the scarring of duodenal ulceration and is believed to be due to cicatrization and adhesions forming consequent upon the inflammatory process in and around the pancreas. However, deformity of the cap can be attributed to underlying pancreatic disease only after the exclusion of duodenal ulceration by means of a negative history of ulcer dyspepsia, a low or normal level of acid secretion, a negative response to ulcer therapy and, if these are equivocal, by the absence of duodenal ulceration shown at laparotomy and indeed at pyloroduodenostomy. The acceptance of such cases as duodenal ulcers would clearly result in an exaggerated incidence of the condition in pancreatitis. (b) The association of peptic ulceration and pancreatitis must inevitably be influenced by the incidence in a given series of localized and clinically unsuspected pancreatitis consequent upon posterior penetration of a peptic ulcer into the pancreas. The proportion of such cases, understandably, varies in different series. Eyler et al. (1962), for example, found a $100 \%$ incidence of duodenal ulceration in a series of 30 patients with pancreatitis, but this remarkable incidence was culled from the surgical records of a group of patients explored because of cholecystitis or duodenal ulceration. A clinical diagnosis of pancreatitis before operation was made in very few, if any, of the patients and in the vast majority the pancreatitis was secondary to penetration of the ulcer itself or to involvement of the pancreas by the inflammatory process surrounding the duodenal ulceration' (Eyler, 1965). The inclusion of a relatively large proportion of such patients in a given series will, we believe, give an erroneous impression regarding the association of peptic ulceration with clinical pancreatitis. (c) The patient material differed from that in most other studies in that it was dominated by patients with alcohol-induced pancreatitis, mainly young or middle-aged males. The incidence of peptic ulceration in the gallstone pancreatitis and miscellaneous groups was no greater than in the alcohol-induced group, however, and it is unlikely that the results could be explained on this basis.

It may be argued that the low incidence of peptic ulceration found in the present study conflicts not only with the clinical experience of previous workers but also with the experimental evidence of others. Pancreatic ablation or obstruction of the pancreatic ducts has been shown to increase acid secretion in the experimental animal (Dragstedt, 1943; Greenlee, Nelsen, and Dragstedt, 1959; Landor and Adams, 1962), but it should be remembered that this is a situation which seldom pertains in patients with even the most advanced pancreatic insufficiency. Welbourn's (1965) recent findings that the inadvertent sparing of a minor pancreatic duct prevented the acid hypersecretory effect of duct ligation in a dog suggests that trivial amounts of pancreatic secretion into the bowel will suffice to prevent the acid hypersecretory effect of pancreatic duct ligation or pancreatic ablation. On the other hand, the elegant study carried out by Zollinger et al. (1962) in a patient with chronic pancreatitis associated with intractable peptic ulceration, suggests that the well-documented experimental observations may indeed have a clinical counterpart. However, the failure to find a similar case of intractable peptic ulceration in the present series, and the demonstration by Kravetz and Spiro (1965) and Bank, Marks, and Groll (1966) that patients with pancreatitis tend toward a low level of acid secretion, suggests that the elaboration of an acid secretory hormone by the diseased pancreas must be a physiopathological curiosity hardly pertinent to the overall problem of the incidence of peptic ulceration in pancreatitis.

The present paper draws attention to the variety of causes responsible for gastrointestinal haemorrhage in patients during an attack of pancreatitis. While peptic ulceration, gastrooesophageal varices due to cirrhosis, and gastric carcinoma must always be excluded, our findings suggest that bleeding during an attack of pancreatitis is usually related to the effects of alcohol or the pancreatitis itself. These include acute gastric erosions or mucosal congestion secondary to contiguous pancreatitis, splenic vein obstruction due to pancreatic compression, the Mallory-Weiss syndrome, and alcoholic gastritis. The cause could not be established in a number of patients but it was clearly not due to chronic peptic ulceration or carcinoma. The application of splenoportography to the problem of bleeding during an attack of pancreatitis has led Léger and Crismer (1960) and Sarles, Muratore, Sarles, Camatte, Gaini, and Pastor (1964) to the view that the bleeding in such patients is frequently due to gastric varices developing secondary to splenic vein compression or thrombosis. It is probable that the latter would have been diagnosed with greater frequency in the present series had splenoportography been carried out in those patients in whom the cause of bleeding could not be established.

On the other hand, chronic peptic ulceration, carcinoma, and bleeding from varices due to cirrhosis were the most important causes of bleeding in patients with pancreatitis in the intervals between 
acute attacks and in those patients with biochemical or surgically demonstrable pancreatic disease associated with cirrhosis and penetrating duodenal ulceration. Massive bleeding was a serious, and indeed fatal, complication in a number of patients following pancreatic surgery: digestion of a large blood vessel in the wall of a pseudocyst of the pancreas caused the death of one patient and was considered to be responsible for severe haemorrhage in a further three, two of whom succumbed.

A small number of patients in the present series had postprandial epigastric pain reminiscent of an ulcer-type dyspepsia. Radiological evidence of peptic ulceration was lacking in these patients and they tended to respond poorly, if at all, to ulcer medication. The insidious development of a pancreatic cyst was responsible for the dyspepsia in two of the cases, and appeared to be due to the pancreatitis itself in all but one of the remainder; the exception was a patient in whom attacks of gallstone 'colic' were related to meals.

\section{SUMMARY}

The associatiation between peptic ulceration and pancreatitis has been examined in a series of 529 patients with pancreatitis, 99 of whom had painless pancreatic disease. Gastrointestinal haemorrhage or radiological evidence of peptic ulceration was found in about a fifth of the patients who had had attacks of pancreatitis, but the incidence of peptic ulceration was in fact only $3.5 \%$.

Reasons are advanced for the discepancy between the incidence of peptic ulceration in this and in other reported series. Duodenal cap deformities due to pancreatitis but attributed to peptic ulceration were regarded as a possible cause of the discrepancy, as was the inclusion of a relatively large proportion of patients with clinically unimpressive localized pancreatitis secondary to posterior penetration of a peptic ulcer in any given series.

Attention is drawn to the occasional but massive haemorrhage that may occur in patients following drainage procedures on a pancreatic cyst and to the variety of causes responsible for gastrointestinal haemorrhage in patients during an attack of pancreatitis. These include acute gastric erosions or mucosal congestion secondary to contiguous pancreatitis, splenic vein obstruction due to pancreatic compression, the Mallory-Weiss syndrome, alcoholic gastritis and, possibly, salicylate erosions. Chronic peptic ulceration, varices due to cirrhosis, and carcinoma were the major causes of haemorrhage in patients who were not suffering an attack of pancreatitis at the time.
Reference is made to occasional presentation of pancreatitis as an ulcer-type dyspepsia.

We should like to thank Professor P. E. S. Palmer and Dr. L. Werbeloff for advice and encouragement and Dr. J. G. Burger, Superintendent of the Groote Schuur Hospital, for permission to publish.

This study was supported by the South African Council for Scientific and Industrial Research, the Ben May Gastroenterology Research Fund, and the Wellcome Trust.

\section{REFERENCES}

Bank, S., Marks, I. N., and Groll, A. (1966). Gastric acid secretion in pancreatic disease. Gastroenterology, 51, 649-655.

_, - - Moshal, M. G., Efron, G., and Silber, R. (1963). The pancreatic-function test-method and normal values. $S$. Afr. med. J., 37, 1061-1066.

Berk, J. E. (1955). Diagnostic features of pancreatic disease. J. Amer. med. Ass., 159, 1079-1085.

Blumenthal, H. T., and Probstein, J. G. (1959). Pancreatitis-A Clinical Pathologic Correlation. p. 299. Thomas, Springfield, Illinois.

Boyer, J. T., and MacKay, I. R. (1960). The aetiology, course and surgical aspects of pancreatitis. A review of 108 cases. Aust. N. Z.J. Surg., 30, 150-157.

British Medical Journal (1965). Editorial. The Mallory-Weiss syndrome. $2,549$.

Comfort, M. W., Gambill, E. E., and Baggenstoss, A. H. (1946). Chronic relapsing pancreatitis. Gastroenterology, 6, 239-285, 376-408.

Dragstedt, L. R. (1943). Some physiologic problems in surgery of the pancreas. Ann. Surg., 118, 576-593.

Eyler, W. R., (1965). Personal communication.

- Clark, M. D., and Rian, R. L. (1962), An evaluation of roentgen signs of pancreatic enlargement. J. Amer. med. Ass., 181, 967-971:

Farman, J., Werbeloff, L., Marks, I. N., Bank, S., and Louw, J. H. (1966). Proximal duodenal deformities due to pancreatitis. Brit. J. Radiol., 39, 662-668.

Fitzgerald, O., Fitzgerald, P., Fennelly, J., McMullin, J. P., and Boland S. J. (1963). A clinical study of chronic pancreatitis. Gut, 4, 193-216.

Gambill, E. E. (1960). Current practices in general medicine. 14. Acute and chronic relapsing pancreatitis. Proc. Mayo Clin., 35, 67-75.

Greenlee, H. B., Nelsen, T. S., and Dragstedt, L. R. (1959). The effect of pancreatic fistula on gastric secretion. Arch. Surg., 79, 1004-1008.

Howard, J. M., and Jordan, G. L. (1960). Surgical Diseases of the Pancreas, p. 2. Lippincott, Philadelphia.

Konstam, P. (1963). Geographical pathology-West Africa. Clin. Radiol., 14, 206-210.

Kravetz, R. E., and H. M. Spiro, (1965). Gastric secretion in chronic pancreatitis. Ann. intern. Med., 63, 776-782.

Landor, J. H., and Adams, L. A. (1962). Pancreatic duct ligation and gastric secretion. Surg. Forum, 13, 292-294.

Léger, L., and Crismer, R. (1960). Le radiodiagnostic des pancreatitis chroniques. Acta gastro-ent. belg., 23, 396-449.

Lubert, M., and Krause, G. R. (1963). The "ring" shadow in the diagnosis of ulcer. Amer. J. Roentgenol., 90, 767-773.

Marks, I. N., and Bank, S. (1963). The aetiology, clinical features and diagnosis of pancreatitis in the S. Western Cape. S. Afr. med. J., 37, 1039-1053.

Mallory, G. K., and Weiss, S. (1929). Haemorrhages from lacerations of the cardiac orifice of the stomach due to vomiting. Amer. $J$. med. Sci., 178, 506-515.

Sarles, H., Muratore, R., Sarles, J. C., Camatte, R., Gaini, M., and Pastor, J. (1964). Les pancreatites recurrentes. T. Gastro-ent., 7, 11-35.

Warren, K. W., and Cattell, R. B. (1959). Pancreatic surgery. New Engl. med. J., 261, 280-288, 333-340, 387-392.

Welbourn, R. (1965). Personal communication.

Zollinger, R. M., Elliot, D. W., Endahl, G. L., Grant, G. N., Goswitz J. T., and Taft, D. A. (1962). Origin of the ulcerogenic hormon in endocrine induced ulcer. Ann. Surg., 156, 570-578. 